\title{
Condutas sanitárias no enfrentamento da COVID-19
}

\author{
Sanitary conduct in coping with COVID-19 \\ Sanitary conducts not confronted by COVID-19
}

Recebido: 25/04/2021 | Revisado: 03/05/2021 | Aceito: 18/05/2021 | Publicado: 06/06/2021

Kelly Maria Rêgo da Silva

ORCID: https://orcid.org/0000-0003-3070-4060 Laboratório Central de Saúde Pública do Piauí, Brasil

E-mail: kelly-rego@bol.com.br

Márcia Araújo Gualberto

ORCID: https://orcid.org/0000-0001-5102-6241 Hospital São Marcos, Brasil

E-mail:marciagualberto@hotmail.com

Jairo Silva dos Santos

ORCID: https://orcid.org/0000-0001-5650-5305

Hospital São Marcos, Brasil

E-mail: jairo_silsan@hotmail.com

Mariza Gomes

ORCID: https://orcid.org/0000-0002-5050-8924 Hospital São Marcos, Brasil

E-mail: marizamarcia.r.g@gmail.com

Isaias Silva Costa

ORCID: https://orcid.org/0000-0003-2383-5126 Hospital São Marcos, Brasil

E-mail: isaiascostha@hotmail.com

Higo José Neri da Silva

ORCID: https://orcid.org/0000-0003-2089-4147

Universidade Federal do Piauí, Brasil

E-mail: higoneri@gmail.com

Solange Vieira Aquino

ORCID: https://orcid.org/0000-0002-5552-0447

Faculdade Mauricio de Nassau, Brasil

E-mail: solangevi@hotmail.com

Kethellin Miranda Galeno de Carvalho

ORCID: https://orcid.org/0000-0003-3607-6421

Universidade de Nilton Lins, Brasil

E-mail: kethellinrj@gmail.com

Damila Tajra Melo

ORCID: https://orcid.org/0000-0003-0926-0848

Centro Universitário Uninovafapi, Brasil

E-mail: damilatajra@hotmail.com

Anna Letícia de Sousa Marinho

ORCID: https://orcid.org/0000-0002-6021-5981

Centro Universitário Uninovafapi, Brasil

E-mail: lelesmarinho99@gmail.com

Ana Virgínia Marçal do Nascimento

ORCID: https://orcid.org/0000-0002-0321-654X

Especialista em Perícia e Gestão Ambiental, Brasil

E-mail: str.marcal@hotmail.com

Amanda Miranda da Silva

ORCID: https://orcid.org/0000-0001-6476-4809 Universidade Federal do Piaú, Brasil E-mail: miraanda.sa@gmail.com

Renata Sales Abreu Formiga

ORCID: https://orcid.org/0000-0002-1059-3298

Centro de Ensino Unificado de Teresina, Brasil

E-mail: wrenataw@hotmail.com

Fernanda Sales Abreu

ORCID: https://orcid.org/0000-0002-2933-6785

Faculdade Integral Diferencial, Brasil

E-mail: fernandabreu86@hotmail.com

Millena Raimunda Martins de Almeida Carvalho

ORCID: https://orcid.org/0000-0003-2431-2431

Universidade Federal do Piauí, Brasil

E-mail: millenamartinsalmeida2@gmail.com 


\title{
Resumo
}

A medida de proteção mais baratas e extremamente necessária para a não disseminação do vírus é a utilização de equipamentos de proteção individual (como as máscaras), e a higienização adequada, essas precauções valem tanto para ambientes hospitalares quanto domésticos. Este trabalho pretende avaliar artigos que falem da higienização de locais que atendam pacientes com COVID 19, com o intuito de apresentar a importância da higienização e para não contaminação de profissionais, pacientes e acompanhantes para que possa auxiliar de forma positiva na pesquisa. Os dados foram coletados utilizando as bases cientificas: Literatura Latino-Americana e do Caribe em Ciências da Saúde (LILACS), National Library of medicine (PUBMED), PERIODICOS, SCIENCE DIRECT, Biblioteca Virtual em saúde - (BVS), Scientific Eletronic Library Online (SCIELO), Cochrane Library, HighWire Press, Scopus e Elsevier. Foram encontrados um total de 22 artigos (tabela 1) nas bases cientificas: Literatura Latino-Americana e do Caribe em Ciências da Saúde (LILACS), National Library of medicine (PUBMED), PERIODICOS, SCIENCE DIRECT, Biblioteca Virtual em saúde - (BVS), Scientific Eletronic Library Online (SCIELO), Cochrane Library, HighWire Press, Scopus e Elsevier. Foram utilizados como descritores: HIGIENIZAÇÃO, DESCONTAMINAÇÃO, SARS-COV-2, COVID 19 nos idiomas inglês, português e espanhol. Onde obtivemos os artigos abaixo descritos na tabela 1 e discutidos em forma de tópicos relacionados com o tema. Nesse trabalho podemos observar que há uma necessidade da higienização não só para evitar o contágio em pacientes, mas também em profissionais da área. Onde desde o início da pandemia, houve um grande número de casos, fazendo com que eles entrassem para o grupo de risco. Observamos que cada serviço tem uma peculiaridade de higienização e organização de serviços.

Palavras-chave: Higienização; SARS-COV-2; COVID-19; Desinfecção; Saúde.

\begin{abstract}
The cheapest and most necessary protection measure for non-dissemination of the virus is the use of personal protective equipment (such as masks), and proper hygiene, these precautions are valid for both hospital and domestic environments. This work intends to evaluate articles that talk about the hygiene of places that serve patients with COVID 19, in order to present the importance of hygiene and not to contaminate professionals, patients and companions so that it can positively assist in research. Data were collected using the scientific bases: Latin American and Caribbean Literature in Health Sciences (LILACS), National Library of medicine (PUBMED), PERIODICOS, SCIENCE DIRECT, Virtual Health Library - (BVS), Scientific Eletronic Library Online (SCIELO), Cochrane Library, HighWire Press, Scopus and Elsevier. A total of 22 articles (table 1) were found in the scientific bases: Latin American and Caribbean Literature in Health Sciences (LILACS), National Library of medicine (PUBMED), PERIODICOS, SCIENCE DIRECT, Virtual Health Library - (BVS ), Scientific Eletronic Library Online (SCIELO), Cochrane Library, HighWire Press, Scopus and Elsevier. The following descriptors were used: HYGIENIZATION, DECONTAMINATION, SARS-COV-2, COVID 19 in English, Portuguese and Spanish. Where we obtained the articles described below in table 1 and discussed in the form of topics related to the theme. In this work we can see that there is a need for hygiene not only to avoid contagion in patients, but also in professionals in the field. Where since the beginning of the pandemic, there have been a large number of cases, making them join the risk group. We observed that each service has a peculiarity of hygiene and service organization.
\end{abstract}

Keywords: Hygienization; SARS-COV-2; COVID-19; Disinfection; Health.

\section{Resumen}

La medida de protección más económica y necesaria para la no diseminación del virus es el uso de equipos de protección personal (como mascarillas), además de una adecuada higiene, cuidado que es válido tanto para el ámbito hospitalario como doméstico. Este trabajo pretende evaluar artículos que aborden la higiene de los lugares que atienden a los pacientes con COVID 19, con el fin de presentar la importancia de la higiene y no contaminar a los profesionales, pacientes y acompañantes para que pueda ayudar positivamente en la investigación. Los datos fueron recolectados en las bases científicas: Literatura Latinoamericana y del Caribe en Ciencias de la Salud (LILACS), Biblioteca Nacional de Medicina (PUBMED), PERIODICOS, SCIENCE DIRECT, Biblioteca Virtual en Salud - (BVS), Biblioteca Científica Electrónica en Línea (SCIELO), Biblioteca Cochrane, HighWire Press, Scopus y Elsevier. Se encontraron 22 artículos (tabla 1) en las bases científicas: Literatura Latinoamericana y del Caribe en Ciencias de la Salud (LILACS), Biblioteca Nacional de Medicina (PUBMED), PERIODICOS, SCIENCE DIRECT, Biblioteca Virtual en Salud - (BVS), Biblioteca Científica Electrónica en Línea (SCIELO), Cochrane Library, HighWire Press, Scopus y Elsevier. Se utilizaron los siguientes descriptores: HIGIENIZACIÓN, DESCONTAMINACIÓN, SARS-COV-2, COVID 19 en inglés, portugués y español. Donde obtuvimos los artículos descritos a continuación en la tabla 1 y discutidos en forma de temas relacionados con el tema. En este trabajo podemos ver que existe una necesidad de higiene no solo para evitar el contagio en los pacientes, sino también en los profesionales del campo. Donde, desde el inicio de la pandemia, ha habido una gran cantidad de casos, lo que los ha llevado a ingresar al grupo de riesgo. Observamos que cada servicio tiene una peculiaridad de higiene y organización del servicio.

Palabras clave: Higienización; SARS-COV-2; COVID-19; Desinfección; Salud. 


\section{Introdução}

No fim do ano de 2019 ocorreram os primeiros casos do novo coronavírus, esse vírus se tornou uma preocupação para a saúde pública, principalmente por que é de fácil disseminação. Ele é transmitido através das vias aéreas, por isso teve que ser planejado uma estratégia para servir como barreira desse vírus e ainda uma nova organização para melhor auxiliar a população (OPAS, 2020).

O sistema único de saúde (SUS), esse sistema busca ajudar principalmente as pessoas de baixa renda, melhorando a saúde pública no país (CONASS, 2020).

Existem dois tipos de pacientes os sintomáticos (que possuem ou sintomas de forma leve ou complexa) e assintomáticos (pacientes que não possuem sintomas). Os assintomáticos transmitem da mesma forma que os sintomáticos, o que faz com que a doença seja transmitida de forma ainda mais silenciosa e sem controle (ANVISA, 2020).

De acordo com a organização mundial de saúde (OMS) os casos leves são a maioria, ocorrendo em cerca de $80 \%$ dos casos, e apenas $20 \%$ precisam de atendimento hospitalar, por conta da redução de oxigênio (Hopkins, 2020).

De acordo com a ANVISA na nota técnica 07/2020, essa doença é transmitida por gotículas de respiratória ou saliva, quando ocorre uma redução da distância ou algo contaminado.

A medida de proteção mais baratas e extremamente necessária para a não disseminação do vírus é a utilização de equipamentos de proteção individual (como as máscaras), e a higienização adequada, essas precauções valem tanto para ambientes hospitalares quanto domésticos (Miranda, 2020).

Este trabalho pretende avaliar artigos que falem da higienização de locais que atendam pacientes com COVID 19, com o intuito de apresentar a importância da higienização e para não contaminação de profissionais, pacientes e acompanhantes para que possa auxiliar de forma positiva na pesquisa.

\section{Metodologia}

Trata-se de uma pesquisa de revisão integrativa, com objetivo analítico e explicativo com abordagem qualitativa. Essa pesquisa busca através de publicações cientificas descrever seus resultados, explicando suas causas e efeitos. Sua abordagem implica que tudo o que for realizado será qualificado para melhor demonstração dos resultados obtidos pela pesquisa. Onde foi utilizado programa Prisma para melhor distribuição e interpretação dos dados estatísticos (Kauark, 2010; Pereira, 2018).

Foram utilizadas todas as publicações que possuíram dados de higienização e protocolos de limpeza durante a pandemia SARS-CoV-2, publicações cientificas, oficiais da Organização Mundial da Saúde (World Health Organization WHO) e governamentais, dentre a determinada data (2019-2021).

Os dados foram coletados utilizando as bases cientificas: Literatura Latino-Americana e do Caribe em Ciências da Saúde (LILACS), National Library of medicine (PUBMED), PERIODICOS, SCIENCE DIRECT, Biblioteca Virtual em saúde - (BVS), Scientific Eletronic Library Online (SCIELO), Cochrane Library, HighWire Press, Scopus e Elsevier.

Foram analisados os trabalhos publicados e comparados para maior relevância dos resultados e avaliando os dados de higienização e protocolos de limpeza durante a pandemia SARS-CoV-2

Foram utilizados nessa pesquisa todos os trabalhos de higienização e protocolos de limpeza durante a pandemia do novo coronavírus, publicados nos anos de 2019 à 2021, nos idiomas: inglês, espanhol e português. Foram excluídos todos os demais trabalhos e publicações que não se referem ao estudo do novo coronavirus (Covid - 19).

A pesquisa não apresentou nenhum risco, pois todos os dados coletados são ofertados pelas bases cientificas, onde não existiu contato nenhum com os indivíduos infectados e não se fizeram divulgações dos dados pessoais dos indivíduos envolvidos em tais estudos. A pesquisa é voltada a análise de dados para avaliação da higienização e protocolos de limpeza durante a pandemia o novo coronavirus. 


\section{Resultados e Discussão}

Foram encontrados um total de 22 artigos (Tabela 1) nas bases cientificas: Literatura Latino-Americana e do Caribe em Ciências da Saúde (LILACS), National Library of medicine (PUBMED), PERIODICOS, SCIENCE DIRECT, Biblioteca Virtual em saúde - (BVS), Scientific Eletronic Library Online (SCIELO), Cochrane Library, HighWire Press, Scopus e Elsevier. Foram utilizados como descritores: HIGIENIZAÇÃO, DESCONTAMINAÇÃO, SARS-COV-2, COVID 19 nos idiomas inglês, português e espanhol. Onde obtivemos os artigos abaixo descritos na tabela 1 e discutidos em forma de tópicos relacionados com o tema.

Tabela 1. Artigos encontrados e selecionados.

\begin{tabular}{|c|c|c|c|}
\hline \multicolumn{2}{|r|}{ AUTORES } & ARTIGOS & ANO \\
\hline 1 & RALP & $\begin{array}{l}\text { A novel Coronavirus: human-to-human transmission, travel-related } \\
\text { cases, and vaccine readiness. }\end{array}$ & 2019. \\
\hline 2 & $\begin{array}{l}\text { ASSOCIAÇÃO BRASILEIRA DE } \\
\text { MEDICINA DE EMERGÊNCIA }\end{array}$ & $\begin{array}{l}\text { Recomendações para o atendimento de pacientes suspeitos ou } \\
\text { confirmados de infecção pelo novo coronavírus (SARS-cov-2) pelas } \\
\text { equipes de atendimento pré-hospitalar móvel }\end{array}$ & 2020. \\
\hline 3 & MINISTÉRIO DA SAÚDE & $\begin{array}{l}\text { Declara Emergência em Saúde Pública de importância Nacional } \\
\text { (ESPIN) em decorrência da Infecção Humana pelo novo } \\
\text { Coronavírus (2019-ncov). }\end{array}$ & 2020 \\
\hline 4 & GOV UK & COVID-19: guidance for Ambulance Trusts Updated & 2020. \\
\hline 5 & $\begin{array}{l}\text { CENTERS FOR DISEASE } \\
\text { CONTROL AND PREVENTION }\end{array}$ & For COVID-19 in the United States, & 2020. \\
\hline 6 & COSTA & Patient safety culture: evaluation by nursing professionals. $\mathrm{T}$ & 2020 \\
\hline 7 & DAWEI & $\begin{array}{l}\text { Clinical characteristics of 138hospitalized patients with } 2019 \text { novel } \\
\text { coronavirus-infected pneumonia in Wuhan, China. }\end{array}$ & 2020 \\
\hline 8 & DIREÇÃO GERAL DE SAÚDE & COVID-19 & 2020 \\
\hline 9 & YE & $\begin{array}{l}\text { What has been learned and to be learned about the novel coronavirus } \\
\text { disease, }\end{array}$ & 2020. \\
\hline 10 & $\begin{array}{l}\text { WORLD HEALTH } \\
\text { ORGANIZATION }\end{array}$ & Rolling updates on coronavirus disease (COVID-19 & $2020^{\mathrm{a}}$ \\
\hline 11 & NANSHAN & $\begin{array}{l}\text { Epidemiological and clinical characteristics of } 99 \text { cases of } 2019 \text { novel } \\
\text { coronavirus pneumonia in Wuhan, China: a descriptive study, }\end{array}$ & 2019. \\
\hline 12 & $\begin{array}{l}\text { WORLD } \\
\text { ORGANIZATION }\end{array}$ & Report of the WHO-China Joint Mission on Coronavirus Disease & 2020B. \\
\hline 13 & FIGUEIREDO & $\begin{array}{l}\text { Manual de Procedimentos de Higienização e Limpeza para controlo de } \\
\text { infeção nos Serviços de Radiologia da }\end{array}$ & 2020 \\
\hline 14 & $\begin{array}{l}\text { GRUPO DE COORDENAÇÃO } \\
\text { LOCAL DO PROGRAMA DE } \\
\text { PREVENÇÃO E CONTROLO } \\
\text { DE INFEÇÕES E } \\
\text { RESISTÊNCIA } \\
\text { ANTIMICROBIANOS }\end{array}$ & Plano de Contingência COVID-19, & 2020 \\
\hline 15 & SIEMENS & Manual do Operador & 2015 \\
\hline 16 & VARSHNEY & $\begin{array}{l}\text { Initial psychological impact of COVID-19 and its correlates in Indian } \\
\text { Community: An online (FEEL-COVID) survey }\end{array}$ & 2020 \\
\hline 17 & GALLASCH & $\begin{array}{l}\text { Prevenção relacionada à exposição ocupacional do profissional de } \\
\text { saúde no cenário de COVID- } 19\end{array}$ & 2020 \\
\hline 18 & HUN & $\begin{array}{l}\text { To train health personnel to protect themselves from SARS-cov- } 2 \\
\text { (novel coronavirus) infection when caring for a patient or suspected } \\
\text { case. }\end{array}$ & 2020 \\
\hline 19 & LIMA & $\begin{array}{l}\text { Recomendações para cirurgia de emergência durante a pandemia do } \\
\text { COVID-19 }\end{array}$ & 2020 \\
\hline
\end{tabular}




\begin{tabular}{l|l|l|c}
\hline $\mathbf{2 0}$ & OXFORD & Levels of evidence & 2020 \\
\hline $\mathbf{2 1}$ & FERIOLI & $\begin{array}{l}\text { Protegendo os profissionais de saúde da infecção por SARS-cov-2: } \\
\text { indicações práticas }\end{array}$ & 2020 \\
\hline $\mathbf{2 2}$ & CHUGHTAI & $\begin{array}{l}\text { Policies on the use of respiratory protection for hospital health workers } \\
\text { to protect from coronavirus disease (COVID-19) }\end{array}$ & 2020 \\
\hline
\end{tabular}

Fonte: Autores (2021).

\subsection{Higienização no sistema móvel de Urgência}

Assim que a equipe é acionada para o local, ela recebe uma informação detalhada sobre o atendimento que será realizado e a gravidade do paciente, onde o médico irá analisar e conferir a gravidade do paciente. Diversas vezes a equipe não consegue confirmar inicialmente o quadro de saúde. O que se torna necessário uma melhor medida para análise do caso, pois no caso de Covid confirmada o sistema móvel e os profissionais devem ser adequados para os casos (Ralph, 2019).

Uma das mudanças realizadas diferentes de atendimento de outros agravos é a higienização do sistema móvel e equipamentos que agora é realizada pelos próprios profissionais de saúde ou empresa contratada (Associação Brasileira de Medicina de Emergência, 2020).

Essas equipes utilizam detergente neutro, seguida da desinfecção com soluções desinfetantes. Logo após é realizada a desinfecção com álcool 70\% ou hipoclorito de sódio. Após a higienização os panos e utensílios utilizados são descartados em lixo infectante, essas medidas atendem a recomendações da Agência Nacional de Vigilância Sanitária (Anvisa) (Ministério da Saúde, 2020).

Outra medida que foi utilizada foi a proteção de bancos e equipamentos com sacos ou item impermeável grosso para que o que não fosse descartável se tornasse lavável e de fácil higienização (Gov UK, 2020).

Uma outra metodologia adotada foi a montagem de kits (sinais vitais, punção venosa periférica e de intubação orotraqueal), para melhor higienização e evitar contato caso não fosse necessário (Centers for Disease Control and Prevention, 2020).

Na pandemia também está sendo evitado a utilização de ar condicionado na ambulância, outra modificação também foi a utilização de máscara cirúrgica em pacientes do transporte ao local de atendimento. Outro fator também foi a circulação de ar, com a utilização de janelas e exaustor ligados (Costa, 2020).

Toda a parte de anotações, são realizadas após o descarte de equipamentos de proteção individual e utilização de álcool gel. A notificação é realizada pelas unidades fixas hospitalares. Porém ainda existe a necessidade de um melhor preparo das unidades móveis e protocolos que possam especificar as medidas a serem tomadas até de formas mais rápidas (Dawei, 2020).

\subsection{Higienização em salas de Urgência}

O novo vírus nunca havia sido identificado em humanos antes dessa pandemia. Por se tratar de um novo vírus, novos protocolos estão sendo utilizados. Esse vírus possui um período infeccioso de aproximadamente de 7 a 15 dias em média (Direção Geral de Saúde, 2020).

Esse vírus é transmitido de pessoa a pessoa por vias aéreas (por gotículas), objetos contaminados ou transmissão comunitária (onde não se sabe a origem do contágio). Esse vírus pode ficar em superfície por horas até dias, se as superfícies não forem limpas e desinfetadas com frequência. (Ye, 2020).

Pesquisas mostram que o SARS-CoV-2 se pode manter viável em superfícies como plástico ou metal por 72 horas e aerossóis em 3 horas, superfície porosa por 24 horas (Nanshan, 2019). 
Em casas ou lugares públicos, pode se utilizar hipoclorito de sódio ou álcool para higienização, deve ser realizada em grande frequência (Who, 2020A).

Quanto maior a higienização e frequência de limpeza, os riscos são diminuídos. Cada local tem sua padronização de limpeza, principalmente os equipamentos (Who, 2020B).

Os serviços de imagem, por exemplo, existem as áreas sujas são áreas de maior risco de transmissão, onde são realizados procedimentos de risco, exemplo: salas de pequena cirurgia, Salas de Higiene Oral, Salas de tratamento de feridas, Laboratórios e radiologia. Temos também áreas semi-críticas, são áreas de menor risco por terem menos risco de contaminação, são elas: farmácia, ambulatório, salas de vacinação, salas de saúde infantil, gabinetes de consulta (Figueiredo, 2020).

E temos também as áreas não críticas, são áreas de menores contatos como salas de serviços administrativos e similares (Grupo de Coordenação Local do Programa de Prevenção e Controlo de Infeções e de Resistência aos Antimicrobianos, 2020).

Nesse momento crítico de pandemia, é de extrema importância o cumprimento de protocolo de higienização específicos para cada espaço, sendo que deve ser cumprido à risca para diminuição da segurança de pacientes e profissionais da linha de frente (Siemens, 2015).

\subsection{Controle de exposição de pacientes com Covid para redução de contaminação em profissionais de saúde}

A primeira medida de proteção utilizada foi a melhoria na utilização de equipamento de proteção individual como: mascaras, jaleco, óculos, higienização das mãos. A higienização das mãos deve ocorrer antes e depois do contato com o paciente, primeiramente com água e sabão e logo após com álcool 70\% (Varshney, 2020).

Uma outra mudança para os profissionais de saúde foi a construção de fluxos de atendimento e a capacitação, para evitar contaminação ou contágio (Gallasch, 2020).

Mesmo assim, com todas as precauções os profissionais de saúde se tornaram grupo de risco, principalmente os que trabalham na linha de frente, pela quantidade de casos positivos de profissionais de saúde. Outra causa é a sobrecarga de alguns profissionais, falta de descanso, pressão para tratamento e acompanhamento (Hun, 2020).

Todas as salas na entrada devem ter desinfetantes para as mãos 60-95\% de recipientes de álcool e resíduos que podem ser usados sem contato. Também outro ponto que deve ser observado é a distância entre profissionais e paciente, quando possível de $2 \mathrm{~m}$ de distância. Os profissionais devem ter a temperatura monitorada duas vezes por dia (Lima, 2020).

Não é indicado a reutilização de equipamentos de proteção individual ou a utilização de tempo a cima do recomendado pelo fabricante (Oxford, 2020).

Outra mudança que foi realizada foram algumas recomendações para manejo de cirurgias, durante a pandemia, como a obrigatoriedade de um teste negativo para cirurgias eletivas e de urgência (FERIOLI, 2020).

Uma parte a ser considerada é o psicológico dos profissionais, sendo que durante a pandemia houve um aumento de pessoas com depressão, ansiedade e estresse. A fadiga, ameaças e agressões propriamente ditas, perpetradas por pessoas que buscam atendimento e não podem ser acolhidas pela limitação de recursos, e afastamento da família e amigos (Chughtai, 2020).

\section{Considerações Finais}

Nesse trabalho podemos observar que há uma necessidade da higienização não só para evitar o contágio em pacientes, mas também em profissionais da área. Onde desde o início da pandemia, houve um grande número de casos, fazendo com que 
eles entrassem para o grupo de risco. Observamos que cada serviço tem uma peculiaridade de higienização e organização de serviços.

Mediante aos resultados dessa pesquisa, vimos que há uma necessidade maior de criação de padronização de protocolos, para a redução de casos e também de higienização. Necessitamos de mais trabalhos e pesquisas para renovação de dados e uma maior atualização para a população.

\section{Referências}

Associação brasileira de medicina de emergência (ABRAMED). (2020). Recomendações para o atendimento de pacientes suspeitos ou confirmados de infecção pelo novo coronavírus (SARS-CoV-2) pelas equipes de atendimento pré-hospitalar móvel - abril. http://abramede.com.br/wp content/uploads/2020/04/RECOMENDACOES-APH-220420.pdf

Centers for Disease Control and Prevention. (2020). Interim Guidance for Emergency Medical Services (EMS) Systems and 911 Public Safety Answering Points (PSAPS) for COVID-19 in the United States.

Chughtai, A. A., Seale, H., Islam, M. S., Owais, M., \& Macintyre, C. R. (2020). Policies on the use of respiratory protection for hospital health workers to protect from coronavirus disease (COVID-19). Int J Nurs Stud. 105:103567.

Costa, D. B., RAMOS, D., Gabriel C. S., \& Bernardes, A. (2018). Patient safety culture: evaluation by nursing professionals. Texto Contexto Enfermagem [Internet]. http://dx.doi.org/10.1590/0104-070720180002670016

Dawei, W., Bo, H., Chang, H., Fangfang, Z., Xing, L., Jing, Z., et al. (2020). Clinical characteristics of 138hospitalized patients with 2019 novel coronavirusinfected pneumonia in Wuhan, China. JAMA Internal Medicine. https://jamanetwork.com/journals/jama/fullarticle/2761044

Direção Geral de Saúde - DGS. (2020). COVID-19. https://covid19.min-saude.pt em 13/05/2020.

Ferioli, M., Cisternino, C., Leo, V., Pisani, L., Palange, P., \& Nava, S. (2020). Protegendo os profissionais de saúde da infecção por SARS-CoV-2: indicações práticas. Eur Respir R. 29(155): 200068.

Figueiredo, F., (2012). Manual de Procedimentos de Higienização e Limpeza para controlo de infeção nos Serviços de Radiologia da ARS Algarve. Serviços de Radiologia da Administração Regional de Saúde do Algarve.

Gallasch, C. H., Cunha, M. L., Pereira, L., \& Silva-junior, J. S. (2020). Prevenção relacionada à exposição ocupacional do profissional de saúde no cenário de COVID-19. Rev enferm UERJ. 28:e49596.

Gov UK. (2020). Guidance COVID-19: guidance for Ambulance Trusts. https://www.gov.uk/government/publications/covid-19-guidance-for-ambulancetrusts/covid-19-guidance-for-ambulance-trusts

Grupo de Coordenação Local do Programa de Prevenção e Controlo de Infeções e de Resistência aos Antimicrobianos - GCL-PPCIRA. (2020). Plano de Contingência COVID-19, Centro Hospitalar e Universitário de Coimbra. ntranet CHUC

Hopkins, J. (2020). New Cases of COVID-19 In World Countries. University and medicine. https://coronavirus.jhu.edu/data/new-cases

Hun, S. (2020). How to train health personnel to protect themselves from SARS-CoV-2 (novel coronavirus) infection when caring for a patient or suspected case. J Educ Eval Health Prof. 17:10.

Lima, D. S., Filho, J., Gurgel, M. V. S. A., Neto, A. F. A, Costa, E. F. M., Filho, F. X. F. M., et al. (2020). Recomendações para cirurgia de emergência durante a pandemia do COVID-19. J. Health Biol Sci. 8(1):1-3.

Ministério da Saúde (BR). (2020). Brasil confirma primeiro caso de coronavírus 2019. https://www.saude.gov.br/noticias/agencia-saude/46435-brasilconfirma-primeiro-caso-de-novo-coronavirus.

Ministério da Saúde (BR). (2020). Portaria no 188 de 03 de fevereiro de 2020: Declara Emergência em Saúde Pública de importância Nacional (ESPIN) em decorrência da Infecção Humana pelo novo Coronavírus (2019-nCoV). http://www.in.gov.br/web/dou/-/portaria-n-188-de-3-de-fevereiro-de-2020-241408388

Miranda, V. S. G., Rech, R. S., \& Maahs, M. A. P. (2020). Covid-19 edição especial. Revista enfermagem atual in derme. e-020013

Nanshan, C. et al. (2020). Epidemiological and clinical characteristics of 99 cases of 2019 novel coronavirus pneumonia in Wuhan, China: a descriptive study. Lancet. Feb 15; 395(10223):507-513.

Nota técnica gvims/ggtes/anvisa No 07/2020. (2020). Orientações para prevenção e vigilância epidemiológica das infecções por SARS-COV-2 (Covid- 19) dentro dos serviços de saúde.

Organização Pan-americana da Saúde (OPAS). (2020). Folha informativa - COVID-19 (doença causada pelo novo coronavírus). https://www.paho.org/bra/index.php?option=com_content \&view=article\&id=6101:covid19\&Itemid=875.

Oxford. (2009). Centre for Evidence-based Medicine: levels of evidence.

Pereira, A. S., Shitsuka, D. M., Parreira, F. J., \& Shitsuka, R. (2018). Metodologia da pesquisa científica. Universidade Federal de Santa Maria. UAB/NTE/UFSM.1 ${ }^{\text {a }}$ Edição. Santa Maria - RS. 
Research, Society and Development, v. 10, n. 6, e42210615448, 2021

(CC BY 4.0) | ISSN 2525-3409 | DOI: http://dx.doi.org/10.33448/rsd-v10i6.15448

Ralph, R., LEW, J., Zeng, T., Francis, M., Xue, B., Roux, M., et al. (2019). 2019-n CoV (Wuhan virus), a novel Coronavirus: human-to-human transmission, travel-related cases, and vaccine readiness. $J$ Infect.

Siemens. (2015). Manual do Operador - Multitom Rax@, Segurança do Sistema (medidas de segurança do equipamento) - Limpeza e desinfeção, @ Siemens Healthcare $\mathrm{GmbH}$.

Varshney, M., Parel, J.T., Raizada, N., \& Sarin, S. K. (2020). Initial psychological impact of COVID-19 and its correlates in Indian Community: An online (FEEL-COVID) survey. PLoS ONE. 15(5): e0233874.

World Health Organization. (2020a). Rolling updates on coronavirus disease (COVID-19). https://www.who.int/emergencies/diseases/novel-coronavirus2019/events-as-they-happen

World Health Organization. (2020b). Report of the WHO-China Joint Mission on Coronavirus Disease 2019 (COVID-19). https://www.who.int/docs/defaultsource/coronaviruse/who-china-joint-mission-oncovid-19-finalreport.pdf

Ye Yi et al. (2020). COVID-19: what has been learned and to be learned about the novel coronavirus disease. Int J Biol Sci. Mar 15; 16(10): 1753-1766. 